\title{
Emittance exchange in electron booster synchrotron by coupling resonance crossing
}

\author{
J. Kallestrup $\odot^{*}$ and M. Aiba๑ \\ Paul Scherrer Institut, CH-5232 Villigen PSI, Switzerland
}

(Received 4 December 2019; accepted 8 January 2020; published 3 February 2020)

\begin{abstract}
The new generation of storage-ring based synchrotron-radiation light sources imposes challenges on off-axis injection schemes owing to small dynamic apertures. In many upgrade projects of existing light sources, including the Swiss Light Source (SLS) upgrade, it is planned to reuse their injector booster synchrotrons with little to no modifications, although their emittances may not be suitable for the upgraded storage ring. A reduction of injection beam emittance relaxes the requirements for injection devices and machine aperture. Since the beam emittance in an electron synchrotron is much smaller in the vertical plane than in the horizontal, where injections are normally implemented, a significant improvement can be made by reducing the horizontal emittance by exchanging the transverse emittances through coupling resonance crossing. We find a meaningful scaling parameter for the quality of the emittance exchange, showing the relationship between coupling strength and crossing speed. Radiative effects are added to investigate the consequences for electron synchrotrons. Finally, we present the first experimental demonstration of transverse emittance exchange in the SLS booster synchrotron.
\end{abstract}

DOI: 10.1103/PhysRevAccelBeams.23.020701

\section{INTRODUCTION}

Future storage-ring based synchrotron-radiation light sources provide high brilliance photon beams for users by applying the multibend achromat (MBA) lattice concept to the storage ring to reduce the natural emittance. A common side effect of MBA lattices is the small dynamic aperture owing to strong nonlinear magnets required to compensate the large natural chromaticity and higher-order effects.

The small dynamic aperture of the new storage rings limits the usefulness of the traditional off-axis injection scheme used in existing third generation light sources, and injection efficiencies may be significantly below $100 \%$. Possible improvements to the injection may fall into three categories: (1) enlarging the dynamic aperture, (2) making the septum blade thinner, (3) lowering the injection beam emittance. Another solution would be to apply an on-axis injection $[1,2]$ such that the dynamic aperture requirement is significantly relaxed. In this study, we pursue the third option, i.e., lowering the injection beam emittance. In any case, off-axis or on-axis, the smaller emittance will ease injection.

*jonas.kallestrup@psi.ch

Published by the American Physical Society under the terms of the Creative Commons Attribution 4.0 International license. Further distribution of this work must maintain attribution to the author(s) and the published article's title, journal citation, and DOI.
It is preferable for light source upgrade projects to reuse the existing injector complex rather than rebuild a new injector. The last stage of the injector is for most light sources a booster synchrotron [3]. It is also planned to reuse the existing injector complex for the Swiss Light Source upgrade (SLS 2.0) at the Paul Scherrer Institute [4].

One of the approaches to reduce the horizontal emittance is to run the booster off energy, which results in a damping partition shift and consequently a lower horizontal emittance [5]. Another approach is to set the betatron tunes onto the coupling resonance, leading to a redistribution of the natural emittance equally between the horizontal and vertical planes, and has been used for improving injection efficiencies in both proton and electron synchrotrons $[5,6]$. Furthermore, a round-beam operation mode, where the natural emittance is equally distributed in the transverse planes, has gained interest and been tested at synchrotron light source facilities [7,8]. A third method recently proposed is to fully exchange the transverse emittances by swiftly crossing the coupling difference resonance [9]. For electron synchrotrons, the horizontal emittance is generally an order of magnitude larger than the vertical emittance, and therefore it can be decreased drastically through an exchange. The concept of emittance exchange by coupling resonance crossing has previously been observed in the CERN machines ISR, AA, and Proton Synchrotron, while the first systematic study was performed by Carli and Cyvoct in the CERN Proton Synchrotron Booster [10]. In the latter case, the emittance exchange was used in order to measure the horizontal 
emittance in the vertical plane where dispersion is low. We will show how the emittance exchange can be applied to an electron booster synchrotron.

Section II gives simulations of off-axis injections into the SLS 2.0 storage ring to further clarify our motivation of the study. Section III briefly introduces the established theory on emittance exchange by coupling resonance crossing. Furthermore, we investigate the adiabatic conditions together with the effect of synchrotron radiation on the quality of the exchange. In Sec. IV we introduce the SLS booster and present a demonstration of emittance exchange. Our findings are discussed in Sec. V, and we draw our conclusions in Sec. VI.

\section{INJECTION SIMULATIONS}

The main benefit from emittance exchange in the booster is relaxing the storage ring acceptance required for beam injection, especially for horizontal off-axis injection schemes, which are used in most third generation storage rings. We will show the usefulness of emittance exchange for kicker-bump and multipole-kicker injections. We assume a septum thickness of $3 \mathrm{~mm}$ in this section, which is a typical value. As mentioned earlier, a thinner septum is another way to make injection easier. We refer to other studies on that matter $[11,12]$.

\section{A. Kicker bump injections}

The conventional injection scheme is based on an injection septum and a kicker-bump (a series of kicker magnets). The kickers create a temporary orbit bump, bringing the stored beam close to the septum blade. At the same time, the injected beam passes the other side of the septum blade and is, together with the stored beam, brought back toward the nominal orbit, while the kicker magnets are turned off. The injection beam will have a small transverse displacement with respect to the nominal orbit and perform betatron oscillations. Due to synchrotron radiation, the injection beam will slowly damp down toward the nominal orbit and merge with the stored beam. The required horizontal dynamic aperture can be defined as

$$
A_{x}=N_{s} \sigma_{x, s}+2 N_{i} \sigma_{x, i}+S,
$$

where $\sigma_{x, s}$ and $\sigma_{x, i}$ are the horizontal beam size of the stored and injected beam, respectively, and $S$ is the septum blade thickness. $N_{s}$ and $N_{i}$ is the beam clearance in units of $\sigma$ considered for stored and injected beam, respectively. A schematic drawing showing the required aperture is given in Fig. 1. There is an optimum value of $\beta$-function for the injection beam, and thus an arbitrary focusing cannot be applied to make $\sigma_{x, i}$ small. On the other hand, it is obvious that decreasing the horizontal emittance of the injection beam leads to a smaller dynamic aperture required since a smaller injection beam can be placed closer to the stored

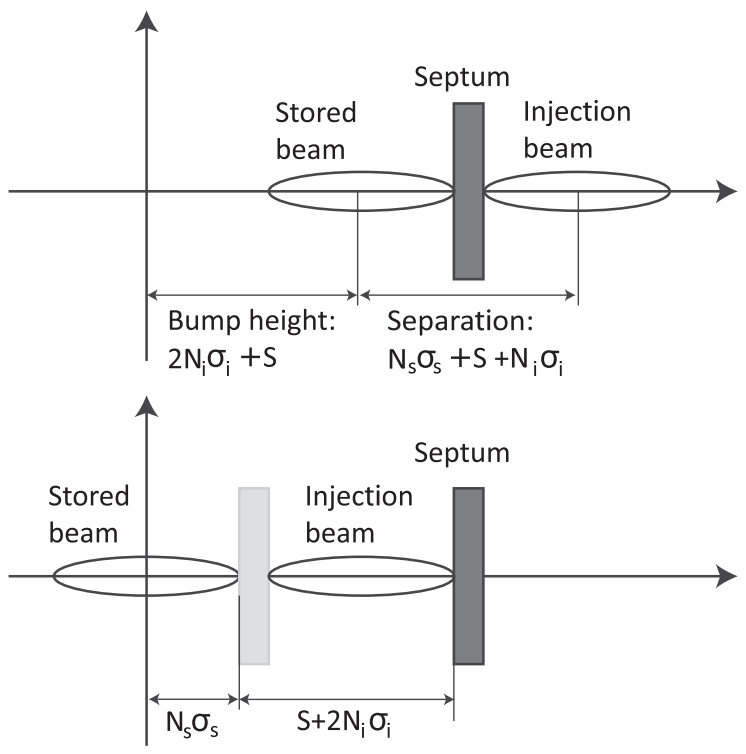

FIG. 1. Schematic showing the setup for the traditional horizontal off-axis injection. (a) Kicker bump on during injection. (b) Kicker bump off after injection. The required horizontal dynamic aperture is given by Eq. (1). Minimizing the horizontal emittance of the injected beam will greatly reduce the required aperture. In this figure, the orbit bump height is minimal: it can be higher if the kicker bump is stronger but not lower.

beam. Figure 2 illustrates the injection into the SLS 2.0 CDR lattice with emittance exchange $\left(\epsilon_{x}=2 \mathrm{~nm} \mathrm{rad}\right.$, $\left.\epsilon_{y}=10 \mathrm{~nm} \mathrm{rad}\right)$ and without $\left(\epsilon_{x}=10 \mathrm{~nm} \mathrm{rad}, \epsilon_{y}=\right.$ $2 \mathrm{~nm} \mathrm{rad}$ ). For the simulations we use $N_{i}=N_{s}=5$ and $S=3 \mathrm{~mm}$. It is seen that the required aperture is decreased from $6 \mathrm{~mm}$ to below $5 \mathrm{~mm}$ because the injected beam centroid can be closer to the stored beam. The lattice used for this simulation does not include any machine imperfections, and the dynamic aperture is slightly more than $6 \mathrm{~mm}$. However, it deteriorates to $5 \mathrm{~mm}$ or less with a typical set of errors [4].

\section{B. Multipole kicker injections}

Another injection scheme that would benefit from a smaller horizontal emittance is based on the usage of multipole kickers as pioneered by KEK-PF [13]. Advanced designs are being developed at many facilities (see, e.g., [14-19]) and one of them is already being used in nominal operation at the MAX IV $3 \mathrm{GeV}$ ring [20]. Here, the injected beam arrives with a transverse offset at a pulsed multipole kicker magnet. The nonlinear field will then give the injected beam a feed-down dipole kick while the stored beam at the kicker axis will be left essentially unperturbed. At the same time, a feed-down quadrupole defocuses the injection beam. The injection efficiency may deteriorate due to the defocusing when the relevant parameters are tight (see, e.g., [21]). A simulated example of the usefulness of emittance exchange in the case of 


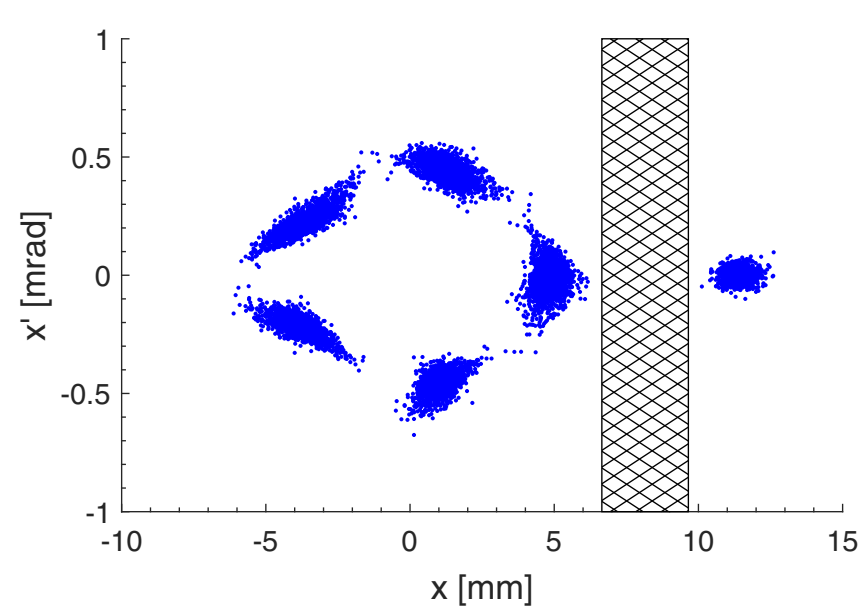

(a)

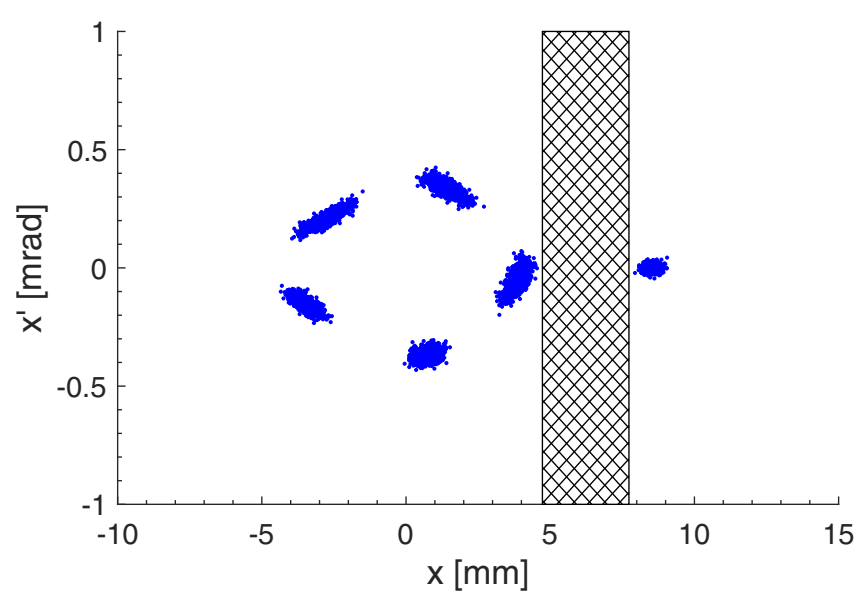

(b)

FIG. 2. Example of horizontal kicker bump injection into the SLS 2.0 storage ring. The first 10 turns in the machine is included. (a) $\epsilon_{x}=10 \mathrm{~nm}$ rad without emittance exchange. (b) $\epsilon_{x}=2 \mathrm{~nm}$ rad with emittance exchange. The required aperture is significantly decreased because the injected beam can be brought closer to the stored beam.

a pulsed sextupole kicker injection example is plotted in Fig. 3. The smear of the injected beam is significantly decreased when emittances are exchanged. The feed-down quadrupole acts on the injected beam also in the vertical plane. The associated vertical emittance growth is not critical since the assumed equilibrium emittance of the booster is rather low in this simulation. For a multipole kicker injection with a large emittance booster, a partial emittance exchange may be applied to properly distribute the equilibrium emittance between the horizontal and vertical planes, maximizing the injection efficiency. The injection beam Twiss parameters can be matched taking into account the feed-down quadrupole of the multipole kicker if the injection beam line has the required capabilities. However, the injection beam size at the septum tends to be larger to compensate for a rather strong feed-down quadrupole.

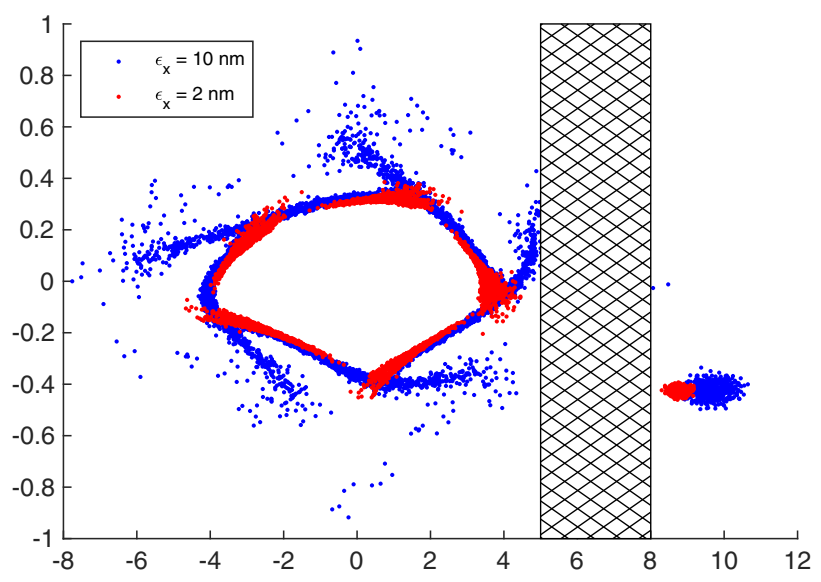

(a)

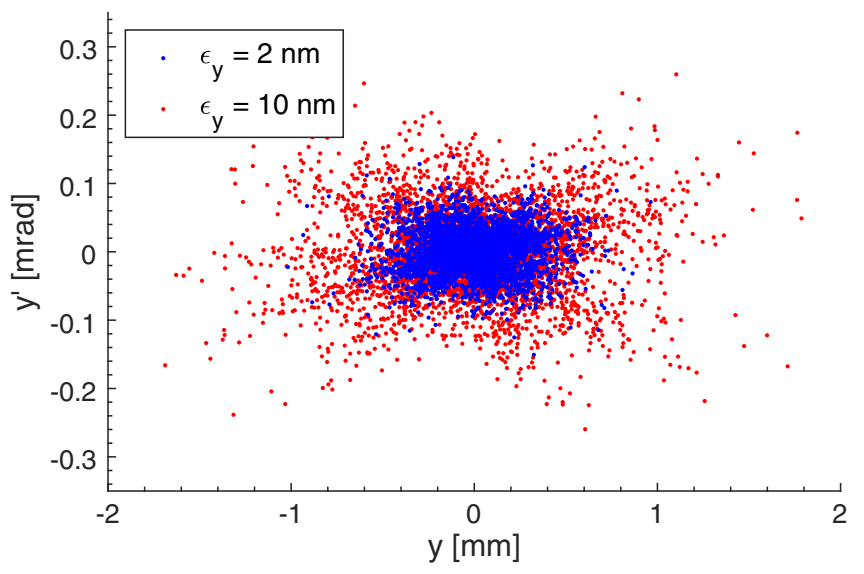

(b)

FIG. 3. Tracking simulations of a multipole kicker injection based on a pulsed sextupole magnet with and without emittance exchange. The first 10 turns in the machine are included. (a) Horizontal phase space (b) vertical phase space. The lower horizontal emittance due to emittance exchange significantly reduces the smear of the injected beam in the horizontal phase space arising from the kicker magnet defocusing and the strong lattice nonlinearity.

\section{THEORY AND SIMULATIONS}

The coupling of the transverse planes can conveniently be quantified by the coupling coefficient, for the difference resonance, as

$$
C=-\frac{1}{2 \pi} \oint d s K_{s} \sqrt{\beta_{x}(s) \beta_{y}(s)} e^{-i\left[\phi_{x}(s)-\phi_{y}(s)+\frac{s}{R} \Delta\right]},
$$

where $K_{s}$ is the skew quadrupole component at location $s$, $\beta_{x}$ and $\beta_{y}$ are $\beta$-functions in the horizontal and vertical planes, respectively, $R$ is the average radius of the machine, and $\Delta$ is the separation of the fractional, uncoupled betatron tunes defined as

$$
\Delta=Q_{x}-Q_{y}-\ell=\hat{Q}_{x}-\hat{Q}_{y},
$$


where $\ell$ is an integer and $\hat{Q}_{x, y}$ are the fractional parts of the tunes. When measuring the tunes of a machine with nonzero coupling, one does not find $\hat{Q}_{x}$ and $\hat{Q}_{y}$. Instead, the normal mode tunes, $Q_{1}$ and $Q_{2}$, are measured due to the finite coupling [22]

$$
Q_{1,2}=Q_{x, y} \mp \frac{1}{2} \Delta \pm \frac{1}{2} \sqrt{\Delta^{2}+|C|^{2}},
$$

with $|C|$ being the amplitude of the coupling coefficient defined in Eq. (2). This equation reveals that the normal mode tunes cannot cross each other; when $\Delta=0$ (i.e., $\hat{Q}_{x}=\hat{Q}_{y}$ ) we have $\hat{Q}_{1}-\hat{Q}_{2}=|C|$, and the normal mode tunes are separated by a finite stop band of size $|C|$. The amplitude of the coupling coefficient can therefore be measured from the minimum separation of the fractional parts of the normal mode tunes by step-wise changing the tunes with quadrupoles. This method is commonly known as the closest-tune approach. We will, for the remainder of this paper, omit the integer part of the tunes such that $Q_{x, y}=\hat{Q}_{x, y}$ for simplicity.

\section{A. Existing treatment}

The effect of resonances on beam emittances is obviously of great interest in synchrotrons. The classical formulas for the sharing of transverse emittance in the presence of a coupling difference resonance was derived by Guignard for the static case, i.e., without crossing [23]. He later included radiative effects in context of the Large Electron-Positron Collider [22].

Following the Proton Synchrotron Booster experimental studies of the emittance exchange by resonance crossing, a theoretical description of the phenomena was developed in [24]. The beam emittance evolution was derived as a function of $|C|$ and $\Delta$, using the angle between the laboratory frame axis and the normal mode axis. This derivation was later improved through the use of the formalism of Hamiltonian resonance driving terms [25]. These descriptions are, however, without radiation damping and for adiabatic crossing and therefore not very useful for our study.

\section{B. Crossing in electron ring}

In this subsection we investigate resonance crossings in electron rings with emphasis on adiabaticity and radiative effects. The simulation results shown here are for the SLS booster (see Sec. IVA for details).

\section{Adiabaticity}

When treating particle motion near resonances, the concept of adiabaticity becomes important [26]. The changes to the emittances must remain adiabatic during the coupling resonance crossing in order to obtain a full exchange. Crossing the resonance too fast will lead to an incomplete exchange. The crossing speed on the other hand is a measure relative to the coupling coefficient: if the coupling coefficient is small, then a long crossing time will be needed to satisfy the adiabaticity constraint.

The evolution of the action-angle coordinates, $J$ and $\psi$, for a coupled system when moving the vertical tune to cross a linear coupling resonance has previously been described by Lee et al. in [27]. The following expressions apply to the actions

$$
\begin{aligned}
\frac{d J_{x}}{d \theta}= & -2|C| \sqrt{Q_{x} Q_{y} J_{x} J_{y}} \cos \theta \sin \psi_{x} \cos \psi_{y}, \\
\frac{d J_{y}}{d \theta}= & -2|C| \sqrt{Q_{x} Q_{y} J_{x} J_{y}} \cos \theta \cos \psi_{x} \sin \psi_{y} \\
& +\frac{\dot{Q}_{y}}{Q_{y}} J_{y}\left(\cos ^{2} \psi_{y}-\sin ^{2} \psi_{y}\right) .
\end{aligned}
$$

A dot refers to the time derivative. The original work includes a damping term for the vertical action, which has been excluded here. Dividing Eq. (5b) by $|C|$ leads to

$$
\begin{aligned}
\frac{d J_{y}}{d \theta} \frac{1}{|C|}= & -2 \sqrt{Q_{x} Q_{y} J_{x} J_{y}} \cos \theta \cos \psi_{x} \sin \psi_{y} \\
& +\frac{\dot{Q}_{y}}{Q_{y}} \frac{J_{y}}{|C|}\left(\cos ^{2} \psi_{y}-\sin ^{2} \psi_{y}\right) .
\end{aligned}
$$

From this expression we find that a parameter $\mathcal{V} \equiv \dot{Q}_{y} /|C|$ (with unit turn $^{-1}$ ), which we define as the normalized crossing speed, is a more generic parameter than $\dot{Q}_{y}$. At the same time, the time step is scaled by $|C|$ on the left-hand side of the equation. Therefore, an interesting scaling parameter for the action is found to be

$$
\mathcal{S}=\frac{\mathcal{V}}{|C|}=\frac{\dot{\Delta}}{|C|^{2}}
$$

Here, $\dot{Q}_{2}$ is replaced by $\dot{\Delta}$ to include general cases, where both tunes are moving. The adiabaticity of the crossing is simply described by the scaling parameter, independent of the accelerator lattice details.

To show that this is indeed the case, we have simulated an emittance exchange with various values of $\mathcal{S}$. $\mathcal{S}$ is changed by modifying $|C|$ and $\dot{\Delta}$, keeping an initial and final value of $|\Delta|=10|C|$. A high value of $|\Delta|$ is preferred to minimize the emittance oscillations induced due to the finite coupling. The results are shown in Fig. 4, where the quality of the exchange is quantified with the ratio $R$ defined as

$$
R=1-\frac{\epsilon_{x, \text { end }}-\epsilon_{y, 0}}{\epsilon_{x, 0}-\epsilon_{y, 0}}
$$




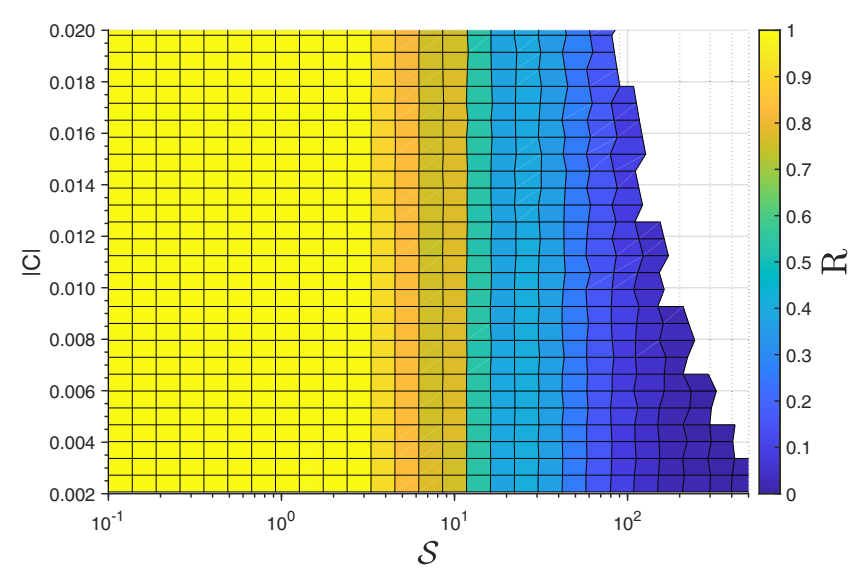

FIG. 4. Quality of emittance exchange as a function of the scaling parameter for the SLS booster lattice.

where $\epsilon_{x, \text { end }}$ is the horizontal emittance after the exchange and $\epsilon_{x, 0}$ and $\epsilon_{y, 0}$ are the horizontal and vertical emittances before the exchange, respectively. A full emittance exchange $\left(\epsilon_{x, \text { end }}=\epsilon_{y, 0}\right)$ corresponds to $R=1$.

An initial particle distribution is created using a random number generator and matched to the lattice. The particles are tracked using linear one-turn maps. Since the coupling is included in the linear map and the particle distribution is bounded for the crossing of linear difference resonance, the nonlinearity of the lattice is not important for our simulations; we checked this by comparing with element-byelement tracking including sextupoles. The one-turn map is modified each turn to include the dynamic process of the resonance crossing. The emittances are evaluated after each turn by calculating the determinant of the covariance matrix of the particle distribution in the laboratory coordinate. For information, all simulations are performed using the Accelerator Toolbox. [28].

The results show that $\mathcal{S}$ is indeed a proper scaling parameter to describe the adiabaticity of the exchange. The quality of the emittance exchange is constant over $|C|$ for a given $\mathcal{S}$. For larger crossing speeds $(\mathcal{S}>3)$ the quality of the exchange quickly drops off, meaning that the crossing has become nonadiabatic. The upper right corner (high $\mathcal{S}$ and high $|C|$ ) is not interesting for us. This is due to the fact that to have a large value of both $\mathcal{S}$ and $|C|$, one must perform the crossing in only a few turns, which is irrelevant at least for booster synchrotrons. From this investigation, we set the first condition to achieve a good quality emittance exchange: $\mathcal{S}<3$.

\section{Radiative effects}

When transverse emittance exchange is performed in an electron synchrotron, the emittances will be out of equilibrium and the synchrotron radiation damping and quantum excitation will lead to a cancellation of the emittance exchange after some time. The effect starts to appear immediately after $\Delta=0$. To show this, we simulate the

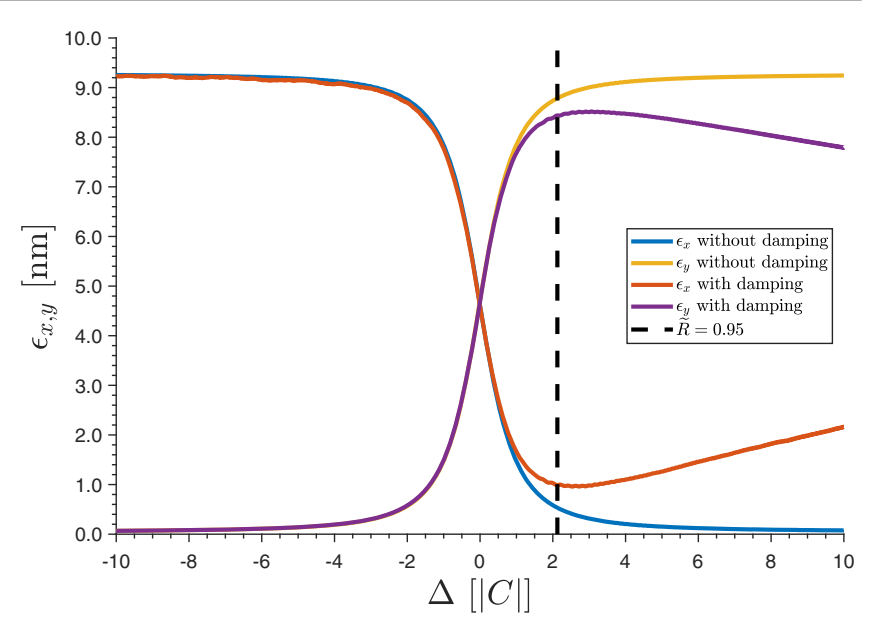

(a)

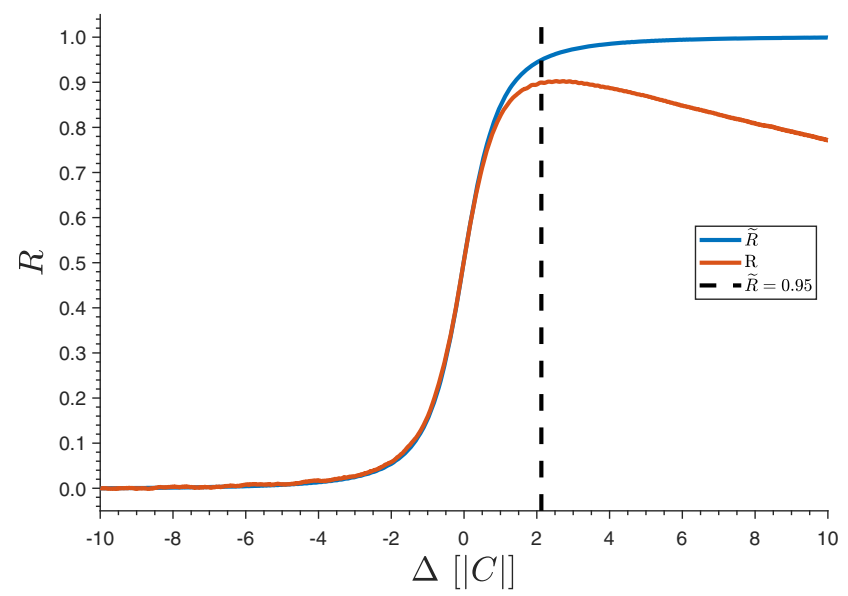

(b)

FIG. 5. Simulated effect of radiation on the emittance exchange process. After the resonance crossing $(\Delta>0)$ the emittances are out of equilibrium, and the radiation damping and quantum excitations will attempt to restore the equilibrium. The dotted line shows the point when $\tilde{R}=0.95$ for the undamped case, which is when $\Delta=2.1|C|$. The simulation is made using the SLS booster lattice using 3400 turns with damping times of $12.7 \times 10^{3}$ turns and $20.6 \times 10^{3}$ turns in the horizontal and vertical plane, respectively, and $\mathcal{S}=0.5$ and $|C|=0.01$.

emittance exchange with and without radiative effects for a parameter set of $|C|=0.01$ and $\mathcal{S}=0.5$, which corresponds to $\approx 3400$ turns. The damping times are $12.7 \times$ $10^{3}$ turns and $20.6 \times 10^{3}$ turns in the horizontal and vertical plane, respectively, for the uncoupled lattice. The results are plotted in Fig. 5.

Due to the radiative effects, the optimum extraction point, where the highest $R$ is achieved, is no longer far away from the resonance. Instead, a compromise between the effect of exchanging emittances and the damping must be found. On the one hand, the emittance exchange has to be almost completed when extracted. On the other hand, the earlier extraction is preferable to minimize the radiative effect. The former sets a condition for the 


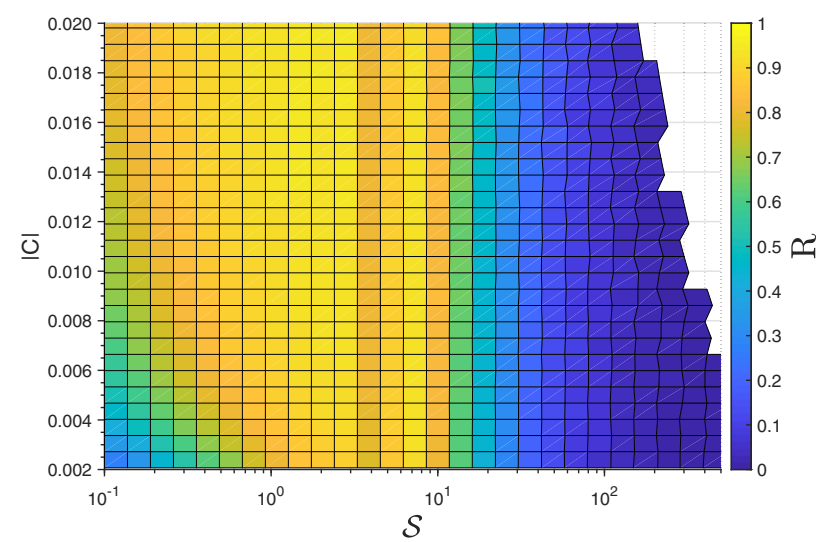

FIG. 6. Quality of emittance exchange as a function of the scaling parameter for the SLS booster lattice including radiative effects. Extraction happens for $\Delta_{\text {ext }}=2.1|C|$.

extraction timing: $\left|\Delta_{\text {end }}\right|>A|C|$ with $A$ being a constant. For instance $A=2.1$ prevents us from extracting the beam too early, as $R$ reaches 0.95 when the damping time is infinitely long. This is the second condition to achieve a quality emittance exchange. The latter will require a selection of parameters and set another condition.

A plot similar to Fig. 4 but this time with radiation and $\Delta_{\text {ext }}=2.1|C|$ is shown in Fig. 6. This plot shows that the region for achieving a good emittance exchange is for $1 \leq \mathcal{S} \leq 3$. The lower cut is due to the radiative effect, whereas the higher cut is due to the adiabaticity constraint.

To further explore the effect of damping (the lower cut), the simulation is repeated for various horizontal damping times and coupling coefficients but for a constant $\mathcal{S}=1$. The damping time is varied by changing the beam energy while keeping the normalized strengths of the lattice magnets. The result is shown in Fig. 7. To achieve a quality emittance exchange, the normalized crossing speed should fulfill

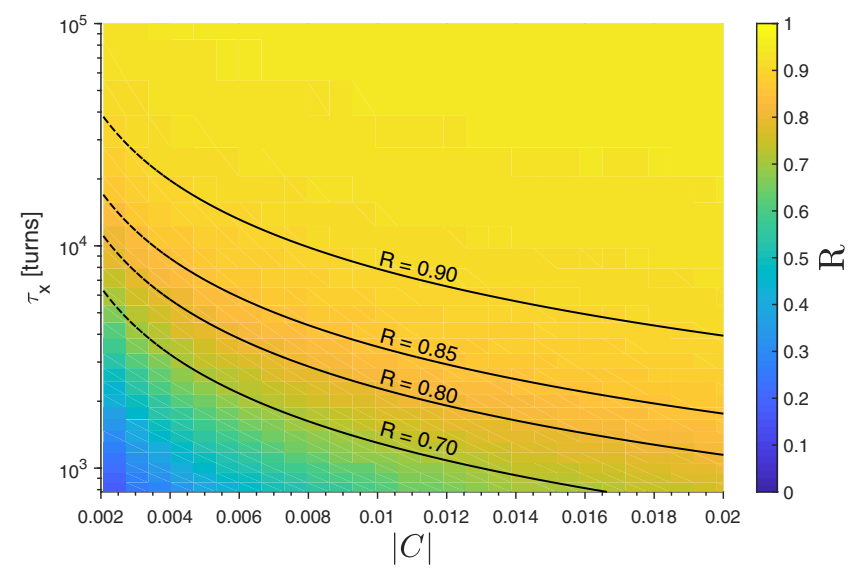

FIG. 7. Quality of emittance exchange for various values of the horizontal damping time and coupling for a fixed value of $\mathcal{S}=1$ and $\Delta_{\text {ext }}=2.1|C|$.

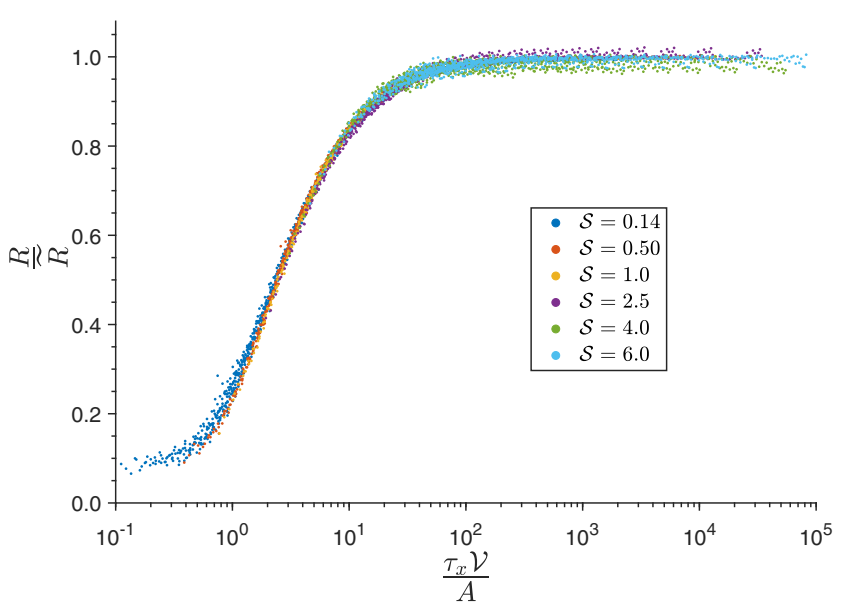

FIG. 8. Deterioration of the emittance exchange due to radiation damping as a function of $\tau_{x} \mathcal{V} / A$. $R$ is divided by $\tilde{R}$, the undamped emittance exchange quality.

$$
\frac{A}{\mathcal{V}}=\frac{A}{|C| \mathcal{S}} \ll \tau,
$$

since $\frac{A}{\mathcal{V}}$ is the time between $\Delta=0$ and the beam extraction. Therefore, we find contours $\tau|C|=$ const. in Fig. 7 where $A$ and $\mathcal{S}$ are constant.

Furthermore, we find that the effect of the damping on the emittance exchange can be generalized. First, we normalize $R$ with the value without radiation damping, denoted by $\tilde{R}$. Second, we normalize the product $\tau \mathcal{V}$, which is found from the inequality Eq. (9), by $A$. The second normalization is based on the fact that $\tilde{R}-R$ is approximately proportional to $A$. This results in approximately a single curve of the normalized $R$ as a function of $\tau \mathcal{V} / A$ (Fig. 8).

From Fig. 8, we can find the last condition to achieve a quality emittance exchange. For instance, $\tau \mathcal{V} / A>30$ achieves a normalized $R$ of about 0.95 . Since the second condition, $A>2.1|C|$, ensures $\tilde{R}>0.95$, the normalized $R$ is approximately (the unnormalized) $R$.

\section{MEASUREMENTS}

\section{A. SLS booster}

SLS pioneered the innovative booster synchrotron design with a circumference similar to the storage ring mounted to the inner tunnel wall with relatively weak and inexpensive magnets. Such a full-scale booster is capable of providing an emittance comparable to the (third generation) storage rings [29]. The design has gained popularity and has since been replicated elsewhere [30-32]. The lattice consists of FODO cells with primary focusing from two combined-function dipole families. Three quadrupole families are placed in the matching section to enable betatron tune adjustment and to suppress dispersion in long straight sections, where the injection septum and rf cavity is located. The dipole families 


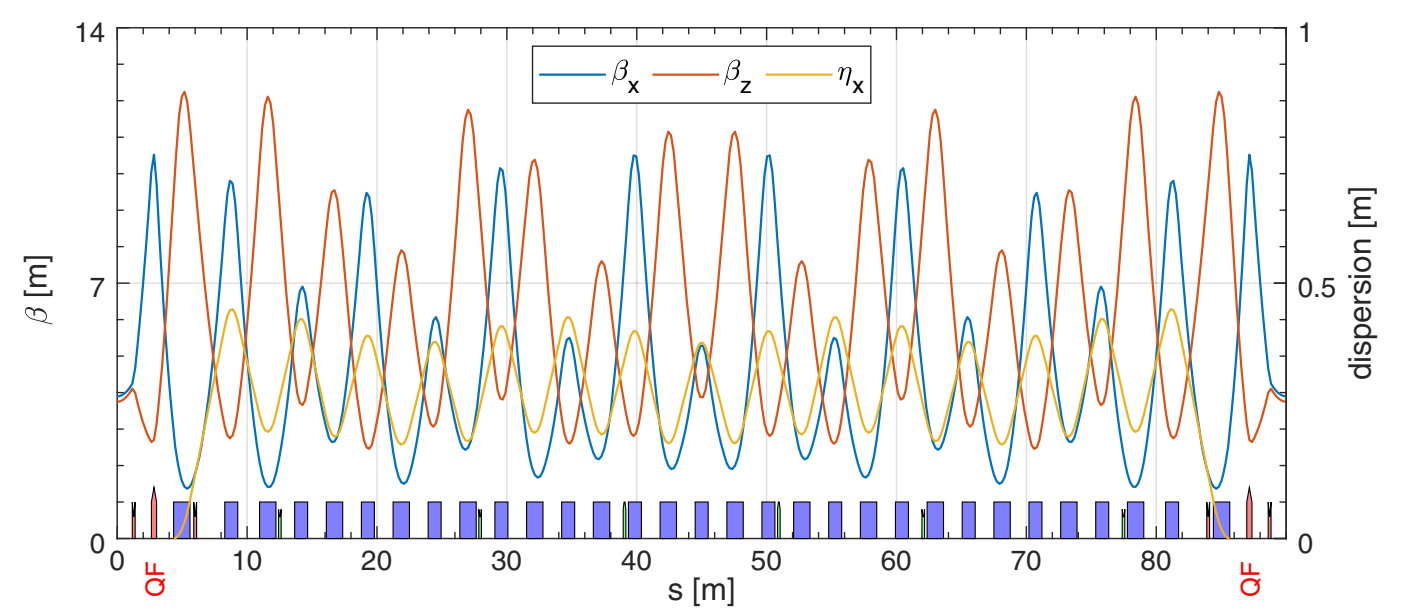

FIG. 9. Optical functions of a SLS Booster superperiod equal to one third of the ring circumference. The regular FODO cell optics is modulated to ease the matching and dispersion suppression into the straight section. The positions of the QF quadrupoles used for tune changes are marked in red.

include a sextupole gradient for a rough chromaticity correction. Two sextupole families are installed for complete correction and compensation of additional sextupole components arising from eddy currents [33]. Optical functions for one superperiod are shown in Fig. 9. The main parameters of the SLS booster are listed in Table I.

Both the SLS storage ring and booster are equipped with digital magnet power supplies. In the case of the booster, this leads to great flexibility compared to boosters using white circuits and enables ad-hoc modifications of the magnet ramping. The full ramping is controllable in 4000 ramp steps; the first 2000 steps define the behavior of the beam before extraction while the remaining 2000 steps are for ramping down. Each ramp step is approximately 88 beam turns, providing good resolution of the optics control along the ramp. Although the horizontal emittance is at the $10 \mathrm{~nm}$ level in the SLS booster, an emittance exchange will still be effective to reduce the horizontal emittance significantly.

TABLE I. Physical SLS Booster specifications and design beam dynamics parameters at $2.4 \mathrm{GeV}$.

\begin{tabular}{lcc}
\hline \hline Injection energy & 0.1 & $\mathrm{GeV}$ \\
Extraction energy & 2.4 & $\mathrm{GeV}$ \\
Circumference & 270.0 & $\mathrm{~m}$ \\
Repetition rate & 3.1 & $\mathrm{~Hz}$ \\
rf frequency & 500 & $\mathrm{MHz}$ \\
No. of superperiods & 3 & $\ldots$ \\
$\epsilon_{x, y}$ & $9.6 / 0.05$ & $\mathrm{~nm} \mathrm{rad}$ \\
$\nu_{x, y}$ & $12.41 / 8.38$ & $\ldots$ \\
Energy spread & $7.5 \times 10^{-4}$ & $\ldots$ \\
Damping time & $11.4 / 18.5 / 13.4$ & $\mathrm{~ms}$ \\
& $12.7 \times 10^{3} / 20.6 \times 10^{3} / 14.9 \times 10^{3}$ & $\mathrm{turns}$ \\
Revolution period & 0.9 & $\mu \mathrm{s}$ \\
\hline \hline
\end{tabular}

\section{B. Booster characterization}

\section{Implementation of resonance crossing}

We first characterized the booster in determine how to implement a coupling resonance crossing. The betatron tune separation, $\Delta$, is of utmost importance for the crossing, and therefore the tunes are measured along the ramp to gain an understanding of the machine under operation conditions. To measure the tunes along the ramp we must excite coherent betatron oscillations. We use the extraction kicker (operating at a low current to apply a reasonable kick to the beam) at different delays to measure the tunes along the ramp. The beam oscillations are measured using a turn-by-turn capable beam position monitor (BPM). A waterfall plot of the FFT spectrum of BPM readings for different times during the ramp can be seen in Fig. 10. The measurements revealed that the working point was close to the coupling shortly after

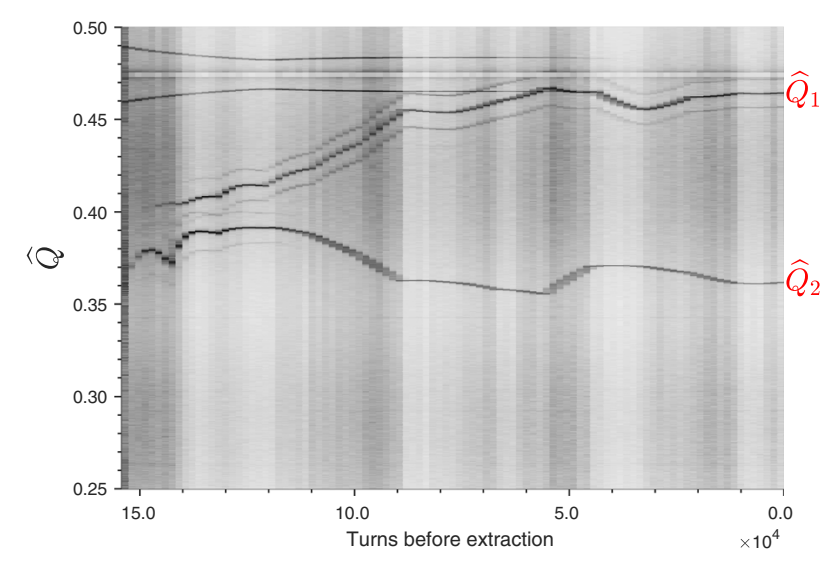

FIG. 10. FFT spectra of turn-by-turn beam position after coherent betatron oscillations are excited by a kicker as a function of turns before extraction. The measured tunes are visible as black lines. 


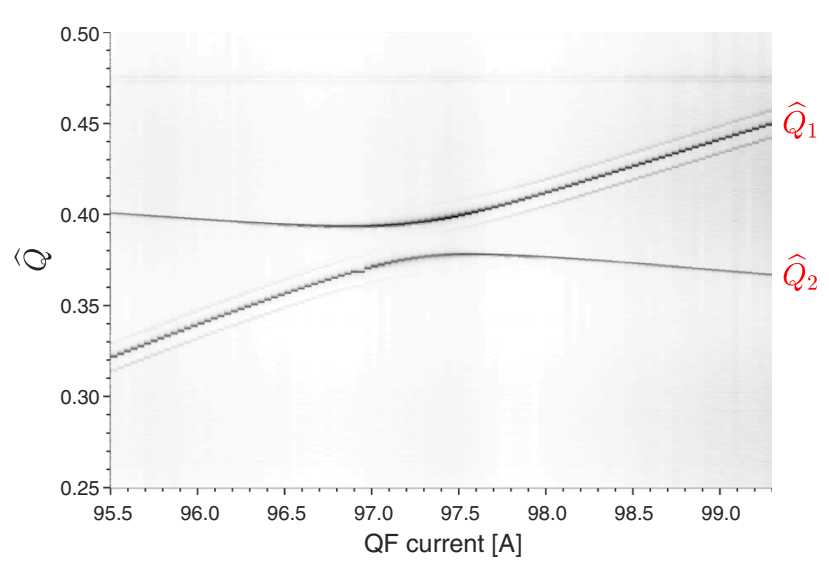

FIG. 11. FFT of beam motion at the top of the ramp for different currents of the QF family. The horizontal fractional normal mode tune changes from 0.325 to 0.450 when changing the QF current from $I_{\mathrm{QF}}=95.5 \mathrm{~A}$ to $99.3 \mathrm{~A}$. The smallest tune separation is reached around $I_{\mathrm{QF}}=97.25$ A revealing a coupling coefficient amplitude of $|C|=0.019$

injection into the booster, while being separated at extraction with fractional tunes $Q_{1}=0.465$ and $Q_{2}=$ 0.365 . Next, we applied a closest-tune approach to measure the coupling coefficient $|C|$ of the booster "as constructed", i.e., including unavoidable machine imperfections. The change in tunes, $\Delta Q$, and change of quadrupole strengths, $\Delta K$, are related as

$$
\Delta K \approx \pm \frac{4 \pi \Delta Q}{\sum_{i} \beta_{i} \ell_{i}},
$$

where $\beta_{i}$ and $\ell_{i}$ refers to the average $\beta$-function over and the length of quadrupole $i$, respectively. The sign depends on the plane (positive for the horizontal plane and negative for the vertical plane), and, similarly, the $\beta_{i}$ is either the horizontal or vertical $\beta$-function. The closest tune can be well measured by varying the quadrupole strength of the QF quadrupole family alone, since the working point of the booster is close to the coupling resonance and the horizontal $\beta$-function at QF quadrupoles is rather high. The betatron tunes were measured for various currents of the QF family at the flat-top. A waterfall plot of the FFT spectra of the beam motion is plotted as a function of QF-family current in Fig. 11. From these measurements we find a coupling coefficient of the booster of $|C|=$ 0.019 .

\section{Implementation of resonance crossing}

Given the preparatory measurements, we use the QF family for a crossing; a resonance crossing was actually achieved during the above closed-tune measurement $\left(I_{\mathrm{QF}}<97.3 \mathrm{~A}\right)$. However, we must properly control the crossing speed, as discussed in Sec. III. Another argument is that, as shown in Fig. 9, QF quadrupoles are located in
TABLE II. QF quadrupole family parameters.

\begin{tabular}{lcc}
\hline \hline No. of magnets & 6 & $\ldots$ \\
Magnetic length & 0.4 & $\mathrm{~m}$ \\
Maximum gradient & 16 & $\mathrm{~T} \mathrm{~m}^{-1}$ \\
Maximum current & 140 & $\mathrm{~A}$ \\
Maximum ramping speed & $1600 / 1.44$ & $\mathrm{~A} \mathrm{~s}^{-1} / \mathrm{mA} \mathrm{turn}^{-1}$ \\
$\beta_{x, y}$ & $10.1 / 2.64$ & $\mathrm{~m}$ \\
$\alpha_{x, y}$ & $-2.67 / 0.286$ & $\ldots$ \\
$\eta_{x, y}$ & $0 / 0$ & $\mathrm{~m}$ \\
\hline \hline
\end{tabular}

nondispersive sections, and thus the dispersion suppression is not spoiled by changing their strengths. Important parameters of the QF family are listed in Table II. The ramping pattern of the QF family is modified according to Fig. 11: We estimate that a quick quadrupole sweep from $I_{\mathrm{QF}}=98$ A down to 95 A would be suitable, i.e., the final tunes are out of the stop band. Before the quick sweep, $I_{\mathrm{QF}}$ is slowly brought to the initial point of 98 A. To ensure that the quadrupole power supplies follow accordingly, we limit the requested ramping speed to $80 \%$ of the maximum value listed in Table II. With this, a crossing is done in approximately $2.4 \mathrm{~ms}$, a factor 4 less than the damping time.

Figure 12 shows the modified QF-family ramp pattern together with the nominal one. The resulting tunes along the ramp are shown in Fig. 13. As expected, we find that the $Q_{1}$ normal mode tune is moved toward $Q_{2}$ before the crossing happens. The measurement does not give a good resolution of the tunes after the crossing; the number of turns available for FFT after the exchange is limited. Therefore we cannot see from Fig. 13 whether the crossing is completed. We are sure, however, that it is the case from the closest-tune measurement and due to the aforementioned $20 \%$ margin to the ramp speed. With these values we
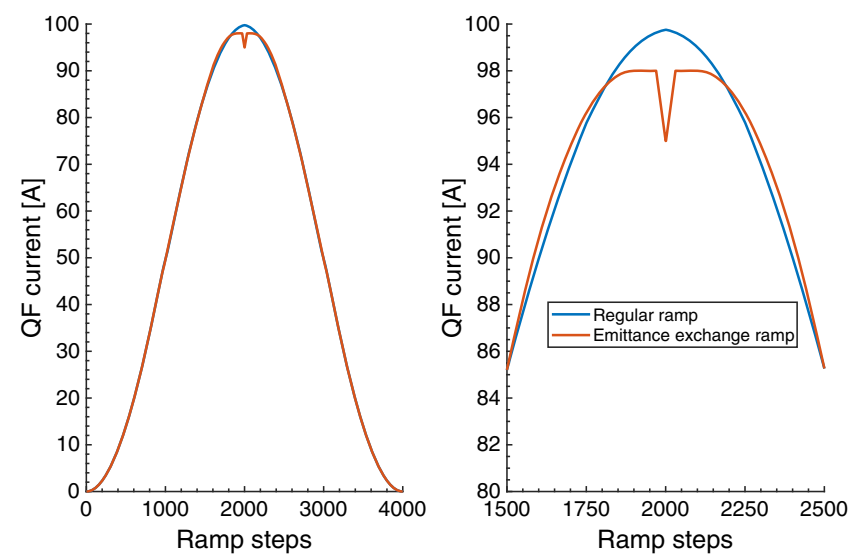

FIG. 12. QF-quadrupole family ramp to achieve emittance exchange. Nominal ramp is also shown for comparison. A short flat-top at $98 \mathrm{~A}$ is followed by a quick drop to $95 \mathrm{~A}$ over the last 30 ramp steps, leading to coupling resonance crossing. Left: Full ramp comparison. Right: Zoom of the modified part of the ramp. 


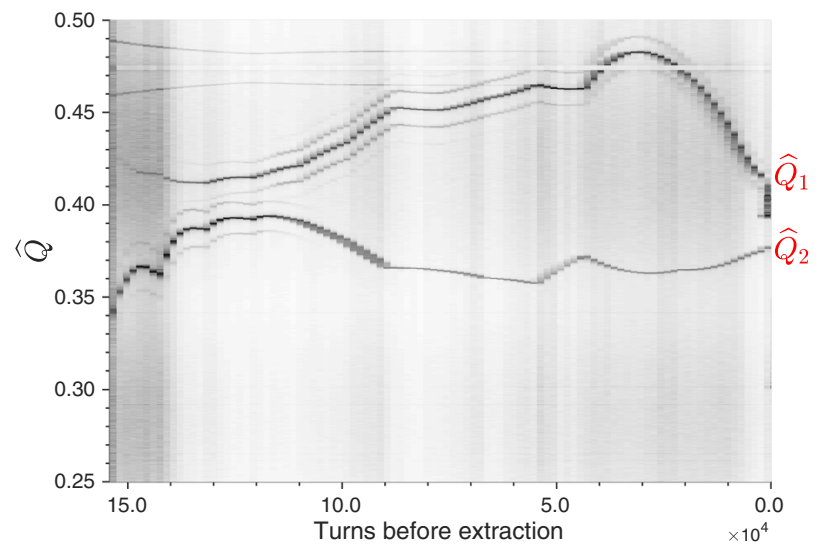

FIG. 13. Tune measurement along booster ramp with emittance exchange. The horizontal tune is brought close to the vertical one during the last $\approx 2500$ turns. The beam is extracted shortly after the coupling resonance crossing to obtain the full emittance exchange.

achieve a scaling parameter of $\mathcal{S} \approx 0.15$, leading to a placement in the upper left corner of Fig. 6. With these parameters we do not expect to achieve a perfect emittance exchange. We discuss this further in Sec. V.

\section{Beam size measurements}

The effect of the emittance exchange is confirmed by measuring how the beam sizes change during the resonance crossing. We extract the beam and measure the beam sizes using an optical transition radiation (OTR) screen in the booster-to-storage ring transfer line. The measurement was performed for various extraction timings to trace the beam sizes as they vary during the crossing. Results are presented in Fig. 14. It is clearly seen that the horizontal beam size decreases whereas the vertical one increases. Since the fractional parts of the tunes are only slightly varied during the crossing, the changes in the booster optics may not be

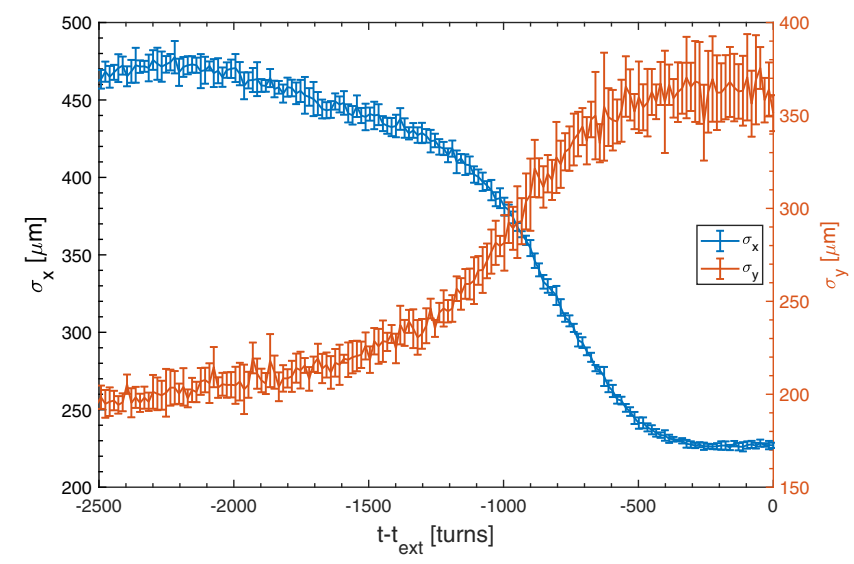

FIG. 14. Beam size measurements for various extraction kicker delays. The beam sizes are measured with an OTR screen installed in the booster-to-ring transfer line. significant. With this proviso, the changes in the beam sizes approximately correspond to the changes in emittances. The ratio of the emittances in either plane before/after the exchange can be found as

$$
\frac{\epsilon_{0}}{\epsilon_{\text {end }}}=\frac{\sigma_{0}^{2}-\left(\eta \frac{\Delta p}{p}\right)^{2}}{\sigma_{\text {end }}^{2}-\left(\eta \frac{\Delta p}{p}\right)^{2}}
$$

where $\epsilon_{0}, \sigma_{0}, \epsilon_{\text {end }}$ and $\sigma_{\text {end }}$ are the emittances and beam sizes before and after the exchange, $\eta$ is the dispersion at the OTR screen and $\frac{\Delta p}{p}$ is the momentum spread. Dispersion is measured by shifting the rf frequency of the booster by $\pm 2 \mathrm{kHz}$, while measuring the displacement of the beam on the OTR screen. The dispersion is then given by

$$
\eta=\frac{\Delta x}{\frac{\Delta p}{p}}=\frac{\Delta x}{\frac{\Delta f_{\mathrm{rf}}}{f_{\mathrm{rf}}} \frac{1}{\alpha_{c}}},
$$

where $\frac{\Delta f_{\text {rf }}}{f_{\text {rf }}}$ is the relative rf frequency shift and $\alpha_{c}$ is the design momentum compaction factor. Inserting the measured beam sizes before and after the exchange together with the measured dispersions and the design values for the momentum spread leads to

$$
\begin{aligned}
& \frac{\epsilon_{x, 0}}{\epsilon_{x, \text { end }}}=4.2, \\
& \frac{\epsilon_{y, \text { end }}}{\epsilon_{y, 0}}=4.4 .
\end{aligned}
$$

The correlation between the decrease in horizontal emittance and increase in vertical emittance is strikingly good: the horizontal decreases by a factor 4.2 while the vertical increases by a factor 4.4 . The results are sensitive to both beam position and size measurements together with the exact value of the energy spread, which in this case is taken to be the design value.

Since the injection efficiency into the SLS storage ring is normally close to $100 \%$, we do not find any effect of the emittance exchange on the injection efficiency.

Another set of beam size measurements, however, has been performed on the beam injected into the storage ring to further confirm the results obtained through the measurements in the transfer line. It is done by measuring the captured beam current as a function of a horizontal scraper position. The results are shown in Fig. 15. It is evident that the horizontal scraper can be inserted much closer to the closed orbit of the stored beam when the emittance exchange is activated. A $Q$-function can be fit to the captured beam current as a function of scraper position:

$$
Q(x)=\frac{1}{2}-\frac{1}{2} \operatorname{erf}\left(\frac{x-\mu}{\sigma_{x} \sqrt{2}}\right),
$$




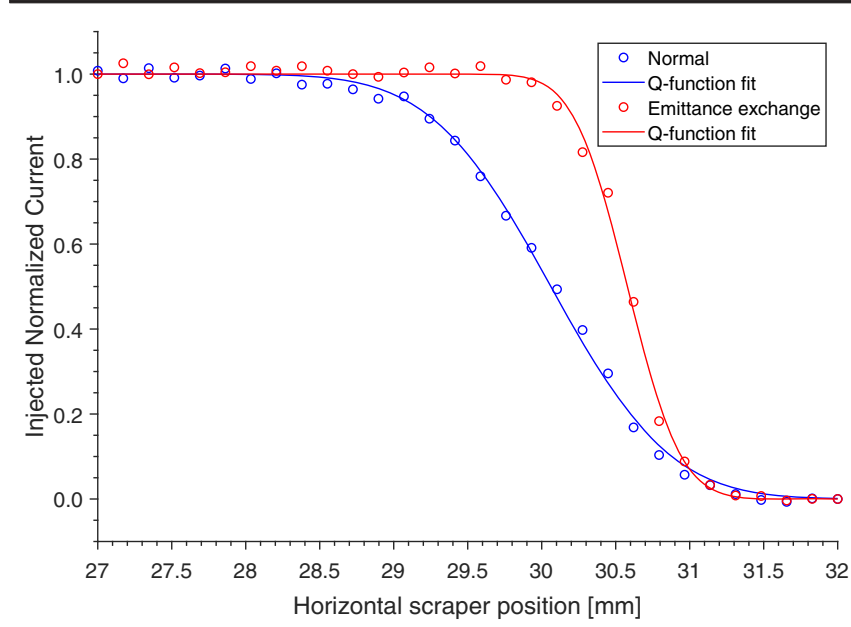

FIG. 15. Measurement of normalized injected current when the horizontal aperture is limited by a scraper in the storage ring. The scraper can be inserted significantly more when the emittance exchange technique is applied.

which leads to an estimate of the horizontal beam size, $\sigma_{x}$, at the scraper position. The ratio between the beam sizes is found as

$$
\frac{\sigma_{x, 0}}{\sigma_{x, \mathrm{EE}}}=2.3
$$

where $\sigma_{x, 0}$ and $\sigma_{x, \mathrm{EE}}$ is the horizontal beam size at the scraper position with and without emittance exchange, respectively. This value is comparable to what was found through the measurement presented in Fig. 14, but cannot be directly compared since the dispersion function at the scraper position has not been measured.

\section{DISCUSSION}

Our studies of the quality of the emittance exchange in Sec. III B revealed that the suggested scaling parameter, $\mathcal{S}$, very well describes the emittance exchange ratio in Eq. (8). The inclusion of radiative effects lead to a reduction of the values of $\mathcal{S}$ and $|C|$ interesting for the practical application of the emittance exchange. We have proposed three conditions to achieve a good emittance exchange, which can be summarized as: (i) $\mathcal{S}<3$, (ii) $A>2.1$, (iii) $\frac{\tau \mathcal{V}}{A}>30$. The first condition requires that the scaling parameter must not be too high, since that would lead to nonadiabaticity of the resonance crossing, and therefore a deterioration of the emittance exchange. Second, the extraction should happen far away from the resonance to ensure a full exchange has been achieved. $\Delta_{\text {ext }}>2.1|C|$ is the value that leads to $R=0.95$, which we define as a good emittance exchange. Finally, the emittance exchange deterioration due to radiation damping is kept to less than $5 \%$ by $\tau \mathcal{V} / A>30$; this together with condition (ii) ensures an emittance exchange quality of $R>0.9$.
The dipole magnets in the SLS booster have a gradient, leading to a damping partition shift. This means that, as the tune separation is decreased, the damping times will also change. At the coupling resonance the transverse damping partition numbers and damping times will become equal, given that the emittance is equalized there. An effect is that Fig. 6, which is calculated for the SLS booster lattice, will be slightly different for other lattices, where the damping partitions are not shifted, and one can assume that $\tau_{\text {before }}=\tau_{\text {after }}$.

Our measurements of the emittance exchange indicate that a near-full emittance exchange was possible in the SLS booster. Accounting for the measured dispersion, we have deduced that the horizontal emittance is decreased approximately by a factor 4 and a similar factor for the vertical emittance increase.

From the closest tune approach measurement we found the scaling parameter of the performed emittance exchange to be $\mathcal{S} \approx 0.15$. With a coupling coefficient of $|C|=0.019$, $\tau V / A \approx 16$ even if we take $\Delta_{\text {ext }}=2.1|C|$, and thus we did not expect a perfect emittance exchange. From the scaling curve (Fig. 8), $R \approx 0.85$. The measured value of $R$ is estimated using the design value for the horizontal emittance and the previously measured value for the vertical emittance, $9.6 \mathrm{~nm}$ and $2 \mathrm{~nm}$ [29], respectively, and the measured ratio of the emittances before/after the exchange. Based on this, we estimate $R=0.96$ of the measured exchange. The result is slightly better than the value that the scaling curve suggested. The difference is attributed to a number of factors. First, the ratio $R$ can be better at $A>2.1$ when the radiation damping time is relatively long. In the experiment, we optimized the extraction time that resulted in the best $R$. Second, the measured value of $R$ is prone to systematic errors in the measurement of the dispersion and the beam size. Finally, the previously measured vertical emittance of the booster may not be an exact value since it was measured only from the beam size assuming the design optics. Exact value of the emittances have not yet been found through the regular quadrupole scan technique due to a relatively high dispersive contribution, both horizontally and vertically, to the beam size. Methods to achieve an accurate measurement are currently under development.

The exchange can potentially be improved in two ways: (1) increasing the quadrupole power supply ramping speed or (2) reducing the coupling coefficient. The second option is more straightforward for the SLS booster. Studies are currently ongoing as to decrease $|C|$ slightly with the expectation of an improved emittance exchange.

The advantage of the emittance exchange was visualized using a horizontal scraper in the storage ring, and seeing how far the scraper could be inserted before injection was deteriorated. With the emittance exchange, the scraper could be inserted significantly further. This measurement showed the applicability of emittance exchange for storage 
rings with small horizontal dynamic aperture and horizontal off-axis injection. This measurement also gave us an estimate of the beam size ratio with and without emittance exchange. In future studies we plan to do the same measurements with vertical scrapers. This measurement is relevant for the usage of minigap undulators.

Several existing light sources plan to upgrade their storage rings in the near future (e.g., [34,35]) while maintaining their existing injector complex with only few modifications to reduce downtime, complexity and costs of the upgrade projects. Their present boosters deliver beam of high ( $\geq 50 \mathrm{~nm} \mathrm{rad}$ ) horizontal emittance (e.g., $[36,37])$, which may restrict their injection efficiencies. By applying emittance exchange through the coupling resonance crossing technique, these facilities might bring down their horizontal emittances by a factor 10 or more, thereby possibly improving the injection efficiency into the upgraded rings and/or relaxing technical requirements and tolerances.

While injection into some storage rings can benefit from a full emittance exchange, others might be limited by a small vertical aperture, either physical or dynamic. The optimum value of $R$ must be found through simulations. The emittance exchange scheme can provide a value of $R>0.5$ by changing the point of exchange along the ramp or the extraction time. If $R<0.5$ is optimum it is, however, better to fix the tunes with a constant $\Delta$ that provides the correct value of $R$.

High-energy boosters, which typically have damping times of a few milliseconds, will require both high coupling and fast quadrupole power supplies to limit deteriorating radiation effects. In those cases, a compromise between the emittance exchange ratio and the technological constraints of the power supply must be found. Another approach is to simply stay at the difference coupling resonance to reach an emittance exchange ratio of $R \approx 0.5$, as done in, e.g., [5,6], thereby imposing no requirements to quadrupole power supplies.

From our studies in Sec. III B 2 of the emittance exchange ratio for a machine including damping we saw that substantial coupling may be needed to perform emittance exchange. If the natural coupling of the booster is not significant, it might be necessary to introduce coupling by installing a skew quadrupole or simply rotating an existing quadrupole.

If the natural machine coupling is low and power supplies are limited in ramping speed, a completely different alternative to the tune coupling resonance crossing is to use a $\pi$-pulsed skew quadrupole magnet as suggested in [9]. Here, the tunes are moved onto the coupling resonance; since little or no coupling is present, there will be no emittance sharing. Emittance exchange is achieved by pulsing the magnet and thereby briefly introducing coupling between the two planes and extracting the beam immediately after.

\section{CONCLUSION}

We have studied transverse emittance exchange by crossing the coupling differences resonance in an electron booster synchrotron, with the goal of relaxing the injection into next-generation storage-ring-based light sources. The requirements on the horizontal dynamic aperture of the storage ring for successful capture of the injected beam are relaxed when the horizontal emittance of the injected beam is decreased. The immediate benefit is shown for two off-axis injection schemes: kicker-bump and multipole kicker injection.

The concept of adiabaticity has been investigated in the frame of the resonance crossing. We have found a scaling parameter for the quality of the emittance exchange that connects the coupling of the machine, the resonance crossing speed and the quality of the emittance exchange. Simulations of emittance exchange including synchrotron radiation and quantum diffusion have been performed. Radiative effects will partly cancel the effect of the emittance exchange, and extraction shortly after the resonance crossing is needed in order not to spoil the emittance exchange. We find a set of general conditions necessary to achieve a quality emittance exchange in electron rings.

Finally, we have successfully demonstrated the concept of emittance exchange by coupling resonance crossing in the SLS booster. The coupling of the machine was measured using the closest tune approach and found to be $|C|=0.019$. With this coupling we did not need to introduce additional skew quadrupole components in the ring. The resonance crossing has been performed by swiftly moving the working point across the coupling difference resonance using one quadrupole family. We found that emittance exchange was indeed possible and a factor 4.2 (4.4) decrease (increase) in horizontal (vertical) emittance was measured. Furthermore, the impact of a smaller horizontal emittance on the injection into aperture-limited storage ring was demonstrated using a horizontal scraper in the SLS.

\section{ACKNOWLEDGMENTS}

The authors thank P. Kuske and M. Böge for useful discussions on the beam dynamics of emittance exchange for electron synchrotrons and coupling resonance crossing. The authors are grateful to C. Özkan for providing technical support for diagnostics during experiments. We would like to thank T. Schietinger for proofreading this manuscript.

[1] M. Borland, A super-bright storage ring alternative to an energy recovery linac, Nucl. Instrum. Methods Phys. Res, Sect. A 557, 230 (2006).

[2] M. Aiba, M. Böge, F. Marcellini, Á. S. Hernández, and A. Streun, Longitudinal injection scheme using short pulse kicker for small aperture electron storage rings, Phys. Rev. Accel. Beams 18, 020701 (2015). 
[3] H. Ohkuma, Top-up operation in light sources, in Proceedings of the 11th European Particle Accelerator Conference, Genoa, 2008 (EPS-AG, Genoa, Italy, 2008), pp. 36-40.

[4] SLS-2 conceptual design report, edited by A. Streun, Paul Scherrer Institute PSI-Report No. 17-03, 2017.

[5] N. Carmignani et al., Operation improvements and emittance reduction of the ESRF booster, in Proceedings of the 9th International Particle Accelerator Conference, IPAC'18, Vancouver, Canada (JACoW Publishing, Geneva, Switzerland, 2018), pp. 4077-4080, https:// doi.org/10.18429/JACoW-IPAC2018-THPMF017.

[6] K. Schindl and P. van der Stok, Increase of betatron stacking efficiency via linear coupling in AG Protron Synchrotrons, CERN Reports No. CERN-PS-BR 76-19, No. CERN-PS-OP 76-5, 1976.

[7] A. Xiao, L. Emery, V. Sajaev, and B. Yang, Experience with round beam operation at the Advanced Photon Source, in Proceedings of the 6th International Particle Accelerator Conference, (IPAC'15), Richmond, USA (JACoW, Geneva, Switzerland, 2015), https://doi.org/ 10.18429/JACoW-IPAC2015-MOPMA013.

[8] Y. Hidaka, W. X. Cheng, Y. Li, T. Shaftan, and G. Wang, Round beam studies at NSLS-II, in Proceedings 6th International Particle Accelerator Conference, (IPAC'15), Richmond, USA (JACoW Publishing, Geneva, Switzerland, 2018), https://doi.org/10.18429/JACoW-IPAC2018TUPMK018.

[9] P. Kuske and F. Kramer, Transverse emittance exchange for improved injection efficiency, in Proceedings of the 7 th International Particle Accelerator Conference, IPAC'16, Busan, Korea (JACoW, Geneva, Switzerland, 2016), pp. 2028-2031, https://doi.org/10.18429/JACoWIPAC2016-WEOAA01.

[10] C. Carli and C. Cyvoct, Emittance exchange by crossing a coupling resonance: A method for precise measurement of the horizontal emittance applied to the PSB, CERN Report No. PS-AE-Note 2001-018, 2001.

[11] D. Shuman et al., Stray field reduction in ALS eddy current septum magnets, in Proceedings of the 21st Particle Accelerator Conference, Knoxville, TN, 2005 (IEEE, Piscataway, NJ, 2005).

[12] C. H. Gough and M. Aiba, Top-up injection with antiseptum, in Proceedings of the 8th International Particle Accelerator Conference, IPAC'17, Copenhagen, Denmark (JACoW, Geneva, Switzerland, 2017), pp. 774-776, https://doi.org/10.18429/JACoW-IPAC2017-MOPIK104.

[13] H. Takaki et al., Beam injection with a pulsed sextupole magnet in an electron storage ring, Phys. Rev. Accel. Beams 13, 020705 (2010).

[14] T. Atkinson, M. Dirsat, O. Dressler, and P. Kuske, Development of a nonlinear kicker system to facilitate a new injection scheme for the BESSY II Storage Ring, in Proceedings of the 2nd International Particle Accelerator Conference, San Sebastián, Spain (EPS-AG, Spain, 2011), pp. 3394-3396.

[15] T. Pulampong and R. Bartolini, A non-linear injection kicker for Diamond Light Source, in Proceedings of the 4th International Particle Accelerator Conference, IPAC-
2013, Shanghai, China, 2013 (JACoW, Shanghai, China, 2013), pp. 2268-2270.

[16] C. Pappas et al., Development of nonlinear injection kicker magnet for ALS accelerator, in Proceedings of the 6th International Particle Accelerator Conference, (IPAC'15), Richmond, USA (JACoW, Geneva, Switzerland, 2015), pp. 1837-1839, https://doi.org/10.18429/JACoWIPAC2015-TUPJE084.

[17] L. Liu, X. Resende, A. Rodrigues, and F. de Sá, Injection dynamics for Sirius using a nonlinear kicker, in Proceedings of the 7th International Particle Accelerator Conference, (IPAC'16), Busan, Kora (JACoW, Geneva, Switzerland, 2016), pp. 3406-3408, https://doi.org/ 10.18429/JACoW-IPAC2016-THPMR011.

[18] P. Alexandre, Development and operation of a multipole injection kicker for SOLEIL and MAX IV, in Presented at 2nd Topical Workshop on Injection and Injection Systems, Villigen, Switzerland (2019) [https://indico.psi.ch/event/ 6972/].

[19] R. Auchettl and Y.-R. E. Tan, Proposed nonlinear injection kicker for the Australian Synchrotron, in Proceedings of the 10th International Particle Accelerator Conference, IPAC'19, Melbourne, Australia (JACoW Publishing, Geneva, Switzerland, 2019), pp. 23002303, https://doi.org/10.18429/JACoW-IPAC2019WEPMP001.

[20] P. F. Tavares et al., Commissioning and first-year operational results of the MAXIV $3 \mathrm{GeV}$ ring, J. Synchrotron Radiat. 25, 1291 (2018).

[21] S. C. Leemann, Pulsed sextupole injection for Sweden's new light source MAX IV, Phys. Rev. Accel. Beams 15, 050705 (2012).

[22] G. Guignard, Betatron coupling and related impact of radiation, Phys. Rev. E 51, 6104 (1995).

[23] G. Guignard, Beam blow-up and luminosity reduction due to linear coupling, CERN Report No. CERN-ISR-BOM77-43, 1977.

[24] E. Métral, Simple theory of emittance sharing and exchange due to linear betatron coupling, CERN Report No. CERN-PS-2001-066 (AE), 2001.

[25] A. Franchi, E. Métral, and R. Tomas, Emittance sharing and exchange driven by linear betatron coupling in circular accelerators, Phys. Rev. Accel. Beams 10, 064003 (2007).

[26] A. W. Chao and M. Month, Particle trapping during passage through a high-order nonlinear resonance, Nucl. Instrum. Methods 121, 129 (1974).

[27] M. J. Lee, E. D. Courant, C. Pellegrini, and A. M. Sessler, Beam amplitude behavior upon crossing a linear coupling resonance with damping in one dimension, IEEE Trans. Nucl. Sci. 16, 176 (1969).

[28] A. Terebilo, Accelerator Modeling with MATLAB Accelerator Toolbox, in Proceedings of the 19th Particle Accelerator Conference, Chicago, IL, 2001 (IEEE, Piscataway, NJ, 2001).

[29] W. Joho, M. Muñoz, and A. Streun, The SLS booster synchrotron, Nucl. Instrum. Methods Phys. Res, Sect. A 562, 1 (2006).

[30] G. Benedetti, D. Einfeld, M. Muñoz, and M. Pont, Optics for the ALBA booster synchrotron, in Proceedings of the 
11th European Particle Accelerator Conference, Genoa, 2008 (EPS-AG, Genoa, Italy, 2008), pp. 2148-2150.

[31] H. C. Chao et al., Conceptual design of booster synchrotron for TPS, in Proceedings of the 11th European Particle Accelerator Conference, Genoa, 2008 (EPS-AG, Genoa, Italy, 2008), pp. 2981-2983.

[32] F. De Sá, L. Liu, X. R. Resende, and A. R. D. Rodrigues, A new booster synchrotron for the Sirius project, in Proceedings of the 5th International Particle Accelerator Conference, IPAC'14, Dresden, Germany (JACoW Publishing, Geneva, Switzerland, 2014), pp. 1959-1961, https:// doi.org/10.18429/JACoW-IPAC2014-WEPRO009.

[33] M. Muñoz and W. Joho, Eddy current effects in the SLS booster, Paul Scherrer Institute Report No. SLS-TMETA-1998-0010, 1998, http://slsbd.psi.ch/pub/slsnotes/tmeta 9810/eddy.html.
[34] E. Karantzoulis, Elettra 2.0, the diffraction limited successor of elettra, Nucl. Instrum. Methods Phys. Res, Sect. A 880, 158 (2018).

[35] A. Loulergue et al., Baseline lattice for the upgrade of SOLEIL, in Proceedings of the 9th International Particle Accelerator Conference, IPAC'18, Vancouver, Canada (2018), pp. 4726-4729, https://doi.org/10.18429/JACoWIPAC2018-THPML034.

[36] M. Svandrlik, Overview of the status of the Elettra booster project, in Proceedings of the 11th European Particle Accelerator Conference, Genoa, 2008 (EPS-AG, Genoa, Italy, 2008), pp. 2201-2203.

[37] A. Loulergue, Status of the SOLEIL booster synchrotron, in Proceedings of the 21st Particle Accelerator Conference, Knoxville, TN, 2005 (IEEE, Piscataway, NJ, 2005). 